\title{
Carotid-artery cochlear dehiscence
}

\author{
Vanessa Ribeiro Orlando Galli*, Renata Victória Tassara², \\ Marcelo Castro Alves de Sousa', Mírian Cabral Moreira de Castro', \\ Oswaldo Laércio Mendonça Cruz ${ }^{3}$
}

\begin{abstract}
Changes in course of the internal carotid artery (ICA) are uncommon, and dehiscence of the carotid canal with cochlea may occur. A 48-year-old female individual with pulsatile tinnitus. No other otologic symptoms observed. Otolaryngologic examination and audiometric test with normal results. Computed tomography (CT) scan of the mastoid bones showed dehiscence of cochlea with ICA on the right side. An option for monitored observation was made after analysis of the risks and undefined results of surgery. Patient maintained clinical and audiometric profile. Carotid-artery cochlear dehiscence is a condition that must be known, remembered and investigated, because it may mimic other otologic pathologies. Knowledge about it prevents serious complications that can be difficult to reverse.
\end{abstract}

Keywords: carotid artery; cochlea; dehiscence; pulsatile; tinnitus.

\section{Introduction}

de Belo Horizonte, MG, Brasil

${ }^{2}$ Faculdade Ciências Médicas de Minas Gerais (FCMMG), Belo Horizonte, MG, Brasil

3Universidade Federal de São Paulo (UNIFESP), Departamento de Otorrinolaringologia e Cirurgia de Cabeça e Pescoço, São Paulo, SP, Brasil

Financial support: None. Conflicts of interest: No conflicts of interest declared concerning the publication of this article. Submitted: April 22, 2019. Accepted: September 14, 2019. The study was carried out at the Hospital Santa Casa de Misericórdia de Belo Horizonte e Universidade Federal de São Paulo.

Copyright Galli et al. This is an Open Access article distributed under the terms of the Creative Commons Attribution License, which permits unrestricted use, distribution, and reproduction in any medium, provided the original work is properly cited.
Changes in the course of the internal carotid artery (ICA) in the petrous portion of the temporal bone are uncommon. In general, the so-called petrous carotid presents an upward and vertical path from its final cervical portion, within a bony canal initially formed by the tympanic part of the temporal bone. It is positioned anteriorly to the tympanic and cochlear cavity, and more superiorly medial to the Eustachian tube.

The minimum medial distances to the basal turn (BT) of cochlea of the carotid canal, facial canal (FC) and roof of the jugular fossa were $1.38 \pm 0.82$, $1.06 \pm 0.46$ and $4.68 \pm 2.21 \mathrm{~mm}$, respectively. Carotid canal thinning on the anterior cochlear wall was observed in six (10\%) cases. Fine bone separation was observed between cochlea and FC $(0.1 \mathrm{~mm})$ in one case $(1.67 \%)^{1}$.

In this intratemporal course, the ICA may present bone dehiscence in approximately $1 \%$ of the cases, and positioning and location changes are even rarer $^{1-3}$.

Dehiscence of the bony canal that separates the ICA from other anatomic structures can occur in any portion along its course, including the carotid-cochlear bony plate. When this occurs, contact between the arterial wall and cochlear endosteum, in addition to its exposure to the tympanic cleft, can mimic otologic diseases such as otosclerosis, superior semicircular canal dehiscence syndrome, glomus tumors, and dehiscent jugular bulb4. Although clinical expression depends on the position of dehiscence and on how atypical the carotid canal is, the most common symptom is pulsatile tinnitus occasionally associated with conductive hearing loss ${ }^{1}$. 


\section{Case report}

A 48-year-old female patient complained of pulsatile tinnitus and right-side hearing loss for several years. Hearing loss was only perceived during physical activity, when tinnitus seemed more intense as a result of increased heart rate. She did not report spontaneous dizziness, however, felt dizzy after the Valsalva maneuver. She had no history of head injury or ear surgery and no comorbidities. Otorhinolaryngologic and paired cranial nerve examinations showed normal results. Duplex scan of the carotid and vertebral arteries showed no significant changes. Pure-tone and vocal audiometry showed mild conductive hearing loss on the right side and presence of stapedius reflexes. Computed tomography (CT) scan of the temporal bone showed bone dehiscence with the right carotid canal, maintaining communication with the BT of cochlea - Figure 1. As the symptoms did not significantly interfere with the patient's quality of life, and in view of the risks and undefined results of a possible surgical procedure, an option was made for monitored observation. Currently, the patient is in stable clinical and audiometric conditions.

\section{Discussion}

As previously mentioned, carotid-artery cochlear dehiscence may mimic various otological changes, such as superior semicircular canal dehiscence syndrome, aberrant course of internal carotid artery, glomus tumors, dehiscent jugular bulb, and otosclerosis.

In this case, pulsatile tinnitus, which is typical of this anatomical variation of the carotid artery, can be explained by transmission of the carotid pulse to the cochlea. Conductive hearing loss with stapedius reflexes suggests that this overlap of the cochlear endosteum and the internal carotid artery, with transmission of the carotid pulse to the perilymph, may behave as a third-window syndrome.

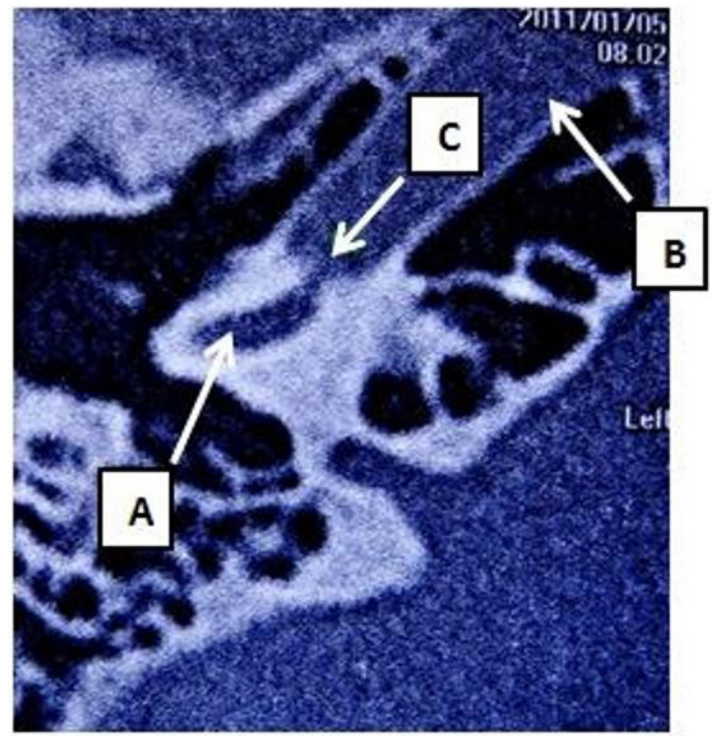

Figure 1. Computed tomography (CT) scan of the temporal bone showing bone dehiscence with the right carotid canal A) basal turn (BT) of cochlea ; B) internal carotid artery (ICA); C) Dehiscence of the carotid canal with the BT. 


\section{*Correspondence}

Vanessa Ribeiro Orlando Galli

Hospital Santa Casa de Misericórdia

de Belo Horizonte

Avenida Francisco Sales 1111, Santa

Efigênia

CEP 30130-110, Belo Horizonte (MG),

Brasi

Tel.: +55 (31) 32388100

E-mail: vanessa.rib.o@gmail.com

\section{Authors information:}

VROG - Médico otorrinolaringologista

e Otologista, mestrando da

Universidade Federal de São Paulo

(UNIFESP); RVT - Acadêmico de medicina da Faculdade de Ciências

Médicas de Minas Gerais; MCAS

- Medico otorrinolaringologista, otologista, mestre em cirurgia pela Universidade Federal de Minas Gerais; MCMC - Médico Otorrinolaringologista doutora em cirurgia pela Universidade Federal de Minas Gerais, coordenadora da clínica de otorrinolaringologia e cirurgia de cabeça e pescoço da Santa Casa de Belo Horizonte; OLMC - Médico Otorrinolaringologista, Otologista, Doutor em Medicina pela Universidade de São Paulo, Professor da Universidade Federal de São Paulo e coordenador do centro de deficiente auditivo da UNIFESP-HSP.
In addition, this type of anatomical change, especially canal bony dehiscence, may pose significant surgical risk during otologic procedures ${ }^{1-4}$. In general, middle ear surgeries are common, safe procedures, but always subject to complications, especially when anatomical changes such as ectopia and carotid-artery cochlear dehiscence are not previously recognized. A possible complication previously reported is the inadvertent introduction of bundles of electrodes into the carotid canal during cochlear implant surgery3,4.

The ICA is very peculiarly arranged in its petrous portion because its outer layer is in direct contact with the bony portion of the canal. Thus, the risk of injury is high upon rupture of this bone canal or of the dehiscent arterial wall $^{5}$. Therefore, rigorous preoperative evaluation is necessary, and it should consider the anatomical relationships between the carotid canal, eardrum, and cochlea $a^{3,4}$.

There are few treatment options for carotid-artery cochlear dehiscence. Considering the complexity of this change, clinical observation may be the best option whenever symptoms do not interfere with the patient's quality of life ${ }^{1,3}$. In cases of severe symptoms, surgical or chemical labyrinthectomy has already been mentioned. Another option would be the balloon occlusion of the ICA, but the risks may outweigh the benefits in such cases ${ }^{4}$.

Petrous carotid artery dehiscence is an uncommon condition, but it may lead to clinical conditions of different aspects. It should also be remembered and investigated prior to otologic surgical procedures, preventing serious complications that can be difficult to reverse.

\section{References}

1. Sahni D, Singla A, Gupta A, Gupta T, Aggarwal A. Relationship of cochlea with surrounding neurovascular structures and their implication in cochlear implantation. Surg Radiol Anat. 2015;37(8):913-9. http://dx.doi.org/10.1007/ s00276-015-1442-7. PMid:25663082.

2. Gibelli D, Cellina M, Gibelli S, Belloni E, Oliva AG, Termine G, Dolci C, Sforza C. Anatomic characteristics of intrapetrous carotid artery: a 3-dimensional segmentation study on head computed tomography scan. World Neurosurg. 2019;121:e419-25. http://dx.doi.org/10.1016/j.wneu.2018.09.125. PMid:30266708.

3. Lund AD, Palacios SD. Carotid artery-cochlear dehiscence: a review. Laryngoscope. 2011;121(12):2658-60. http://dx.doi.org/10.1002/lary.22391. PMid:22109767.

4. Van Damme JP, Heylen G, Gilain C, Garin P. Pulsatile tinnitus associated with dehiscent internal carotid artery: an irremediable condition. Auris Nasus Larynx. 2017;44(5):612-5. http://dx.doi.org/10.1016/j.anl.2016.08.008. PMid:27594410.

5. Nevoux J, Loundon N, Leboulanger N, Roger G, Ducou Le Pointe H, Garabedian, EM. Cochlear implant in the carotid canal. Case report and literature review. International Journal of Pediatric Otorhinolaryngology 74(2010) 701-3. 